\title{
Serological testing for SARS-CoV-2 antibodies in clinical practice: a comparative diagnostic accuracy study
}

\author{
Michael Horn ${ }^{1}$, Hulda R. Jonsdottir ${ }^{2}$, Daniel Brigger ${ }^{2}$, Lauro Damonti ${ }^{3}$, Franziska \\ Suter-Riniker $^{3}$, Olga Endrich ${ }^{1}$, Tanja Fröhlich ${ }^{1}$, Martin Fiedler ${ }^{1}$, Carlo Largiadèr ${ }^{1}$, Jonas \\ Marshall $^{3}$, Benjamin Weber ${ }^{4}$, Alexander Eggel ${ }^{2}$, and Michael Nagler ${ }^{1}$ \\ ${ }^{1}$ Inselspital Universitatsspital Bern \\ ${ }^{2}$ Inselspital Bern Universitatsklinik fur Rheumatologie Immunologie und Allergologie \\ ${ }^{3}$ Universitat Bern Institut fur Infektionskrankheiten \\ ${ }^{4}$ Labor Spiez
}

August 3, 2021

\begin{abstract}
Background: Serological tests are a powerful tool in the monitoring of infectious diseases and the detection of host immunity. However, manufacturers often provide diagnostic accuracy data generated through biased studies and the performance in clinical practice is essentially unclear. Objectives: We aimed to determine the diagnostic accuracy of various serological testing strategies for (a) identification of patients with previous coronavirus disease-2019 (COVID-19) and (b) prediction of neutralizing antibodies against SARS-CoV-2 in real-life clinical settings. Methods: We prospectively included 2'573 consecutive health-care workers and 1'085 inpatients with suspected or possible previous COVID-19 at a Swiss University Hospital. Various serological immunoassays based on different analytical techniques (enzyme-linked immunosorbent assays, ELISA; chemiluminescence immunoassay, CLIA; electrochemiluminescence immunoassay, ECLIA; lateral-flow immunoassay, LFI), epitopes of SARS-CoV-2 (nucleocapsid, N; receptor-binding domain, RBD; extended RBD, RBD+; S1 or S2 domain of the spike [S] protein, S1/S2), and antibody subtypes (IgG, pan-Ig) were conducted. A positive real-time PCR test from a nasopharyngeal swab was defined as previous COVID-19. Neutralization assays with live SARS-CoV-2 were performed in a subgroup of patients to assess neutralization activity $(\mathrm{n}=201)$. Results: The sensitivity to detect patients with previous COVID-19 was [?]85\% in anti-N ECLIA (86.8\%) and anti-S1 ELISA (86.2\%). Sensitivity was $84.7 \%$ in anti-S1/S2 CLIA, $84.0 \%$ in anti-RBD+ LFI, $81.0 \%$ in anti-N CLIA, $79.2 \%$ in anti-RBD ELISA, and $65.6 \%$ in anti-N ELISA. The specificity was $98.4 \%$ in anti-N ECLIA, 98.3\% in anti-N CLIA, $98.2 \%$ in anti-S1 ELISA, $97.7 \%$ in anti-N ELISA, 97.6\% in anti-S1/S2 CLIA, 97.2\% in anti-RBD ELISA, and $96.1 \%$ in anti-RBD+ LFI. The sensitivity to detect neutralizing antibodies was [?]85\% in anti-S1 ELISA (92.7\%), anti-N ECLIA (91.7\%), anti-S1/S2 CLIA (90.3\%), anti-RBD+ LFI (87.9\%), and anti-RBD ELISA (85.8\%). Sensitivity was $84.1 \%$ in anti-N CLIA, and $66.2 \%$ in anti-N ELISA. The specificity was [?] $97 \%$ in anti-N CLIA (100\%), anti-S1/S2 CLIA (97.7\%), and anti-RBD+ LFI (97.9\%). Specificity was $95.9 \%$ in anti-RBD ELISA, 93.0\% in anti-N ECLIA, 92\% in anti-S1 ELISA, and $65.3 \%$ in anti-N ELISA. Diagnostic accuracy measures were consistent among subgroups. Conclusions: The diagnostic accuracy of serological tests for SARS-CoV-2 antibodies varied remarkably in clinical practice, and the sensitivity to identify patients with previous COVID-19 deviated substantially from the manufacturer's specifications. The data presented here should be considered when using such tests to estimate the infection burden within a specific population and determine the likelihood of protection against re-infection.
\end{abstract}

\section{ORIGINAL ARTICLE}

Serological testing for SARS-CoV-2 antibodies in clinical practice: a comparative diagnostic accuracy study Michael P. Horn, $\mathrm{PhD}^{1^{*}}$, Hulda R. Jonsdottir, $\mathrm{PhD}^{2,3,8^{*}}$, Daniel Brigger, $\mathrm{PhD}{ }^{2,3}$, Lauro Damonti, $\mathrm{MD}^{4,5}$, 
Franziska Suter-Riniker, $\mathrm{PhD}^{6}$, Olga Endrich, $\mathrm{MD}^{7}$, Tanja K. Froehlich, $\mathrm{PhD}^{1}$, Martin Fiedler, $\mathrm{MD}^{1}$ Carlo R. Largiadèr, $\mathrm{PhD}^{1}$, Jonas Marschall, $\mathrm{MD}^{4}$, Benjamin Weber, $\mathrm{PhD}^{8}$, Alexander Eggel, $\mathrm{PhD}^{2,3}$, Michael Nagler, $\mathrm{PhD}^{1,3}$

1 University Institute of Clinical Chemistry, Inselspital University Hospital, 3010 Bern, Switzerland; ${ }^{2}$ Department of Rheumatology, Immunology, and Allergology, Inselspital University Hospital, 3010 Bern, Switzerland $;^{3}$ Department of BioMedical Research, University of Bern, 3012 Bern, Switzerland; ${ }^{4}$ Department of Infectious Diseases, Bern University Hospital, and University of Bern, 3012 Bern, Switzerland; ${ }^{5}$ Ente Ospedaliero Cantonale, Division of Infectious Diseases, Regional Hospital Lugano, 6900 Lugano, Switzerland; ${ }^{6}$ Institute for Infectious Diseases, University of Bern, 3012 Bern, Switzerland; ${ }^{7}$ Medical Directorate, Inselspital, University Hospital of Bern, 3010 Berne, Switzerland; ${ }^{8}$ Spiez Laboratory, Federal Office for Civil Protection, 3700 Spiez, Switzerland

* contributed equally

Correspondence: Michael Nagler, MD, PhD, MSc; michael.nagler@insel.ch;

phone +4131664 0520; fax +41316324862

Keywords : Infections/*epidemiology/transmission *Disease Outbreaks; spike protein, SARS-CoV-2 [Supplementary Concept]; severe acute respiratory syndrome coronavirus 2 [Supplementary Concept]; COVID-19 diagnostic testing [Supplementary Concept]

Running head: Serological testing for COVID-19

Word counts: text $=3940 ;$ abstract $=417 ;$ References $=38 ;$ Figures $=6 ;$ Tables $=4$

\section{Abstract}

\section{Background}

Serological tests are a powerful tool in the monitoring of infectious diseases and the detection of host immunity. However, manufacturers often provide diagnostic accuracy data generated through biased studies and the performance in clinical practice is essentially unclear.

\section{Objectives}

We aimed to determine the diagnostic accuracy of various serological testing strategies for (a) identification of patients with previous coronavirus disease-2019 (COVID-19) and (b) prediction of neutralizing antibodies against SARS-CoV-2 in real-life clinical settings.

\section{Methods}

We prospectively included 2'573 consecutive health-care workers and 1'085 inpatients with suspected or possible previous COVID-19 at a Swiss University Hospital. Various serological immunoassays based on different analytical techniques (enzyme-linked immunosorbent assays, ELISA; chemiluminescence immunoassay, CLIA; electrochemiluminescence immunoassay, ECLIA; lateral-flow immunoassay, LFI), epitopes of SARS-CoV-2 (nucleocapsid, N; receptor-binding domain, RBD; extended RBD, RBD+; S1 or S2 domain of the spike $[\mathrm{S}]$ protein, $\mathrm{S} 1 / \mathrm{S} 2$ ), and antibody subtypes (IgG, pan-Ig) were conducted. A positive real-time PCR test from a nasopharyngeal swab was defined as previous COVID-19. Neutralization assays with live SARS-CoV-2 were performed in a subgroup of patients to assess neutralization activity $(n=201)$. 


\section{Results}

The sensitivity to detect patients with previous COVID-19 was [?]85\% in anti-N ECLIA (86.8\%) and antiS1 ELISA (86.2\%). Sensitivity was $84.7 \%$ in anti-S1/S2 CLIA, $84.0 \%$ in anti-RBD+ LFI, $81.0 \%$ in anti-N CLIA, $79.2 \%$ in anti-RBD ELISA, and $65.6 \%$ in anti-N ELISA. The specificity was $98.4 \%$ in anti-N ECLIA, 98.3\% in anti-N CLIA, $98.2 \%$ in anti-S1 ELISA, $97.7 \%$ in anti-N ELISA, $97.6 \%$ in anti-S1/S2 CLIA, $97.2 \%$ in anti-RBD ELISA, and $96.1 \%$ in anti-RBD+ LFI.

The sensitivity to detect neutralizing antibodies was [?]85\% in anti-S1 ELISA (92.7\%), anti-N ECLIA (91.7\%), anti-S1/S2 CLIA (90.3\%), anti-RBD+ LFI (87.9\%), and anti-RBD ELISA (85.8\%). Sensitivity was $84.1 \%$ in anti-N CLIA, and $66.2 \%$ in anti-N ELISA. The specificity was [?]97\% in anti-N CLIA (100\%), anti-S1/S2 CLIA (97.7\%), and anti-RBD+ LFI (97.9\%). Specificity was $95.9 \%$ in anti-RBD ELISA, 93.0\% in anti-N ECLIA, $92 \%$ in anti-S1 ELISA, and $65.3 \%$ in anti-N ELISA. Diagnostic accuracy measures were consistent among subgroups.

\section{Conclusions:}

The diagnostic accuracy of serological tests for SARS-CoV-2 antibodies varied remarkably in clinical practice, and the sensitivity to identify patients with previous COVID-19 deviated substantially from the manufacturer's specifications. The data presented here should be considered when using such tests to estimate the infection burden within a specific population and determine the likelihood of protection against re-infection. 


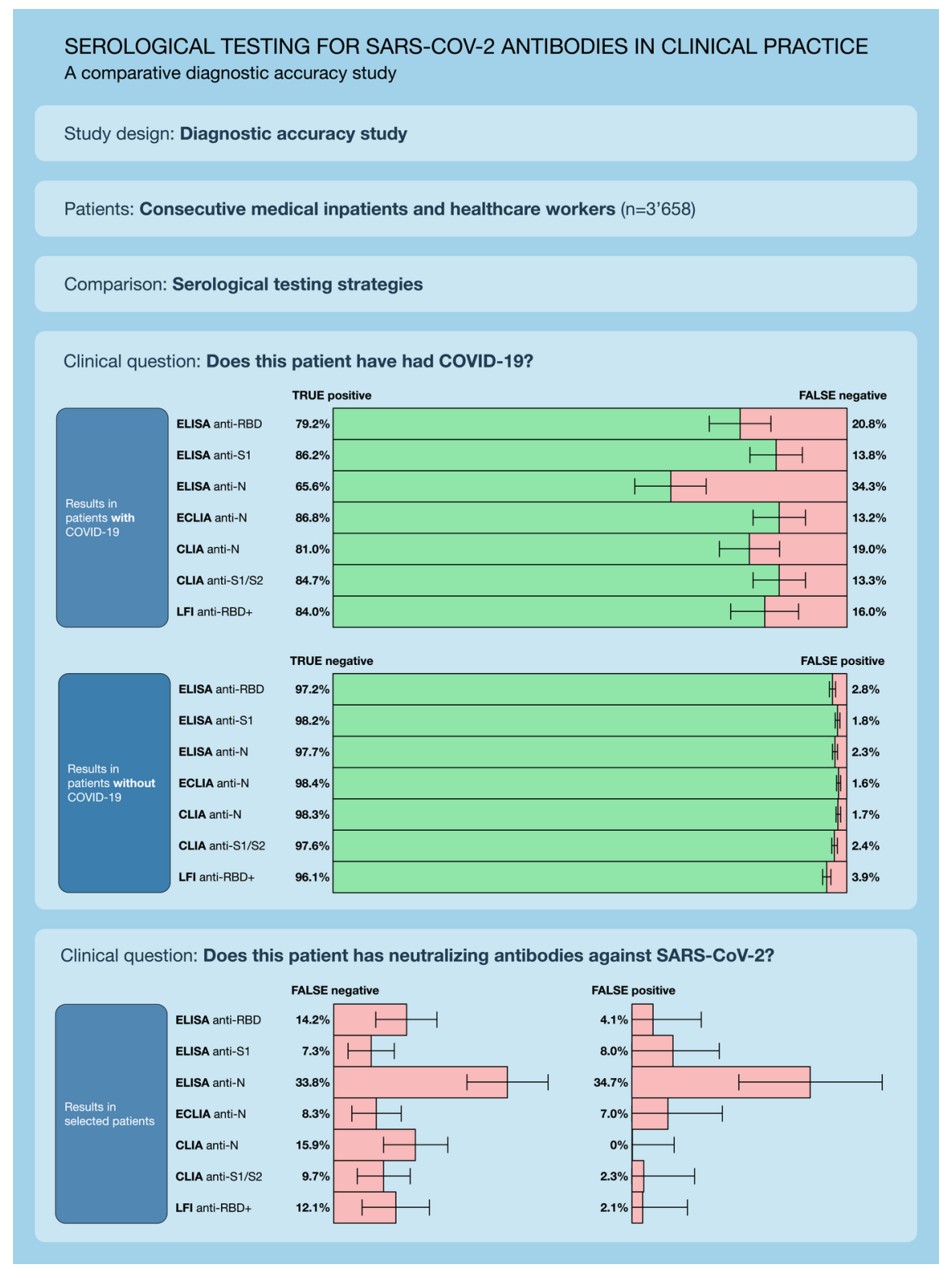

Graphical abstract

\section{Introduction}

Serological tests are a powerful tool in the monitoring of infectious diseases and the detection of host immunity. To help fight the recent coronavirus disease-2019 (COVID-19) pandemic representing our time's sincerest health and socioeconomic crisis, various serological assays have been brought to market in record time. [1-5]. Many of these tests were developed with the ultimate goal to monitor the infection burden within a community, assess vaccination responses, and determine the likelihood of protection against re-infection [5, 6].Broad implementation of serological COVID-19 tests has also been envisioned to assess the effectiveness of control strategies and facilitate decision-making on the reopening of schools, cultural facilities, and businesses [7-10]. Further, such tests might form a basis for the issue of immunity passports, the authorization 
of international traveling, and the return of employees to work $[9,11]$. Numerous serological studies have recently been conducted [12-15], and governments worldwide have ordered millions of serological tests to identify individuals with antibodies against severe acute respiratory syndrome coronavirus 2 (SARS-CoV-2) [16] without prior in-depth clinical validation of the assays.

To enable a meaningful application and interpretation of serological test results, such assays must (a) accurately identify patients with previous COVID-19 and (b) correctly predict protective immunity acquired by previous infection or vaccination $[4,6,10]$. Tests with inadequate performance characteristics will result in misinterpretation of data and might lead to questionable or even counter-productive health policy decision $[5,16]$. Problematically, manufacturers of serological assays often provide diagnostic accuracy data generated through biased studies and claiming to have a sensitivity and specificity close to $100 \%[2,5,12][17][2,10$, $13,18,19]$. Thus, estimates of diagnostic accuracy are regarded as unreliable $[2,10,20]$. Many organizations, including the WHO, now call for the development of reliable antibody tests and evaluation in appropriate diagnostic accuracy studies $[5,9]$.

Here, we conducted a prospective cross-sectional study in a real-life clinical setting, stringently fulfilling the requirements of a diagnostic accuracy study, including (1) an adequately powered prospective design studying clearly defined clinical questions, (2) selection of a representative study population, (3) head-tohead comparison of all significant serological testing strategies, (4) rigorous choice and determination of reference standard, and (e) optimal flow and timing. Specifically, we assessed whether different serological testing strategies may (a) accurately identify patients with previous COVID-19 and (b) correctly predict neutralizing antibodies against SARS-CoV-2.

\section{Methods}

\section{Study design and setting}

The IA-COVID study presented here is a prospective cross-sectional study conducted in a real-life clinical setting at a Swiss University Hospital (Figure 1). First ad interim results have been published previously [21]. Inselspital is the largest tertiary hospital in Switzerland, covering a catchment area of more than 1 million inhabitants (German and French-speaking areas). The study protocol was approved by the appropriate ethical committee (Kantonale Ethikkommission Bern \#2020-00683) and the institutional authorities. All participants signed informed consent. The study was conducted in accordance with the declaration of Helsinki.

\section{Population}

Two groups of individuals representing distinct target populations were included between April and November 2020 (Figure 1): (a) consecutive inpatients with suspected SARS-CoV-2 infection (ill patients at risk for complications), and (b) healthcare workers at Inselspital (healthy individuals tested for surveillance measures). The inclusion criteria for inpatients were: (1) hospitalization in Inselspital, (2) tested at least once for SARS-CoV-2 by real-time polymerase chain reaction (RT-PCR; nasopharyngeal swab), (3) aged 18 or older, and (4) signed informed consent. Only inpatients with more than 4 days of residual sample material were considered. The inclusion criteria for healthcare workers were: (1) medical staff at Inselspital since February 2020, (2) aged 18 or older, and (3) signed informed consent. Healthcare workers were contacted using e-mail lists. A representative subset of patient samples was analyzed with the live SARS-CoV-2 neutralization assay (see below). Additionally, 102 anonymized biobank samples collected from inpatients between December 2018 and February 2019 were analyzed (Liquid Biobank Bern; www.biobankbern.ch). Healthcare workers were contacted by e-mail lists. 


\section{Definition of diagnoses}

We defined a positive RT-PCR from a nasopharyngeal swab as the primary reference standard test representing a "confirmed SARS-CoV-2" infection (COVID-19). We defined "SARS-CoV-2" as negative if (a) RT-PCR was negative in all nasopharyngeal swabs conducted or (b) RT-PCR not performed. Following applicable regulations, all inpatients and medical staff were supposed to be tested in case of symptoms. Biobank samples were classified as negative as they had been collected before the pandemic (i.e., winter 2018/2019).

\section{Handling of samples and collection of data}

To ensure adequate pre-analytical conditions, blood was taken following an established in-house protocol. Samples were collected using plastic syringes containing serum or lithium heparin, respectively (SMonovetteß, Sarstedt, Germany). Residual sample material was used in the case of inpatients; serum and heparin tubes were used in medical staff. Samples were immediately transported to the laboratory and centrifuged within 30 minutes using an established protocol [22].

Pseudonymized demographical, clinical, and laboratory data were extracted from the patient documentation and transferred by the Insel Data Science Center (IDSC) [23]. A REDCap database survey was constructed to collect data of medical personnel (demographic, symptoms, comorbidities, and risk factors).

\section{Selection of immunoassays}

To study all major serological testing strategies, we selected seven immunoassays with different analytical techniques (enzyme-linked immunosorbent assay, ELISA; electrochemiluminescence immunoassay, ECLIA; chemiluminescence immunoassay; CLIA; lateral flow immunoassay, LFI) and epitopes of the SARS-CoV-2 (receptor binding domain of the spike glycoprotein, RBD; S1/S2 domain of the spike glycoprotein; nucleocapsid, N). Key characteristics of the tests are given in Table 2. Considering the results of our own interim analysis and other publications [21], we decided to include assays capturing IgG and pan-Ig.

An in-house anti-RBD ELISA was developed and validated as previously described [21]. An ELISA capturing antibodies against the S1 domain was employed (Euroimmune AG, Lübeck, Germany). Besides, an ELISA determining antibodies against $\mathrm{N}$ was used (Epitope Diagnostics Inc, San Diego, CA, USA). Three highthroughput tests were selected: a pan-Ig ECLIA capturing antibodies against N (Roche diagnostics, Rotkreuz, Switzerland), an IgG CLIA detecting anti-S1/S2 antibodies (DiaSorin S.p.A., Saluggia, Italy), and an IgG CLIA measuring anti-N antibodies (Abbott Laboratories, Sligo, Ireland). Aiming to use a sensitive and precise LFI, we employed a pan-Ig LFI capturing antibodies against an extended RBD protein, which is suggested to be a superior antigen compared to canonical RBD constructs (BÜHLMANN Laboratories, Schönenbuch, Switzerland) [24].

\section{Detection of SARS-CoV-2 specific antibodies}

Laboratory technicians and researchers were blinded to the RT-PCR results and clinical details. All ELISA measurements were done on a DSX automated ELISA system device (DYNEX Technologies, Chantilly, VA, USA) as previously described; instructions of the manufacturers were followed [21]. For the in-house ELISA, 96-well plates were coated, blocked, washed repeatedly, and secondary polyclonal anti-human IgG was added. Results were measured at OD450-620, and samples with an OD > 0.5 were considered positive. Concerning the anti-S1 ELISA measured at OD450-620, antibody values were expressed as a ratio $\left(\mathrm{OD}_{\text {sample }} / \mathrm{OD}_{\text {calibrator }}\right)$, and ratios above 0.8 were assigned positive. With regard to the anti-N ELISA, measurements were conducted at OD450-620 and the cut-off was calculated by the following formula: $1.1^{*}$ (mean $\left.\mathrm{OD}_{\text {negative control }}+0.10\right)$. 
The anti-N ECLIA was conducted on a Cobas 8000 analyzer (Roche diagnostics, Rotkreuz, Switzerland). The cut-off was calculated based on the calibrator measurements and a cut-off index s/c [?] 1.0 was considered positive [25]. Anti-N CLIA was determined on an Architect i2000SR (Abbott AG, Baar, Switzerland). The cut-off was determined by dividing the measurement of the samples by the mean calibrators result; an index [?] 1.4 was considered positive [26]. The anti-S1/S2 CLIA was determined on a Liaison XL (DiaSorin S.p.A., Saluggia, Italy); arbitrary units [?] 12.0 were classified positive [27].

The anti-RBD+ LFI was conducted as previously described [24]. 10 $\mathrm{L}$ serum was transferred to the application pad and designated chase buffer was added. Results were analyzed after 15 minutes using the Quantum Blue@ Reader (3rd generation; BÜHLMANN Laboratories, Schönenbuch, Switzerland) that measures the test line intensity of the LFI in Grey Values [GVs]. Based on the limit of blank plus four times the standard deviation, samples showing GVs of at least 1.5 are considered positive for SARS-CoV-2 antibodies. For this study, GVs were also measured and evaluated quantitatively.

\section{Detection of SARS-CoV-2 by real-time PCR}

Nasopharyngeal swabs (Copan FLOQSwabs and Copan UTM Viral Transport medium; Copan, Brescia, Italy) were transported at room temperature and stored at $4^{\circ} \mathrm{C}$ until processing. Three different methodologies for nucleic acid testing (NAT) were employed in the study period. A laboratory-developed test (LDT) PCR workflow was utilized [28] and two commercial, fully automated assays (Seegene Allplex 2019-nCoV Assay, Seegene, Seoul, Korea; Roche cobas@ SARS-CoV-2 Assay, Roche Diagnostics, Rotkreuz, Switzerland). Details have been reported previously [21]. RT-PCR was done in clinical practice, and laboratory technicians were not aware of the index test results.

\section{Live SARS-CoV-2 neutralization assay}

A representative subset of patients was selected for analysis using a live SARS-CoV-2 neutralization assay $(\mathrm{n}=201)$. To identify potential differences between immunoassays, we included all complex cases (RTPCR+/anti-S1-; RT-PCR-/anti-S1+; RT-PCR+/asymptomatic patients; anti-S1+/anti-N-; anti-S1-/anti$\mathrm{N}+$ ) and other antibody-positive and negative patients. Technicians and researchers conducting and interpreting the neutralization assay were blinded regarding RT-PCR results, serological test details, and clinical details.

Neutralizing antibodies were determined by the inhibition of virus-induced cytopathic effect (CPE). Methodological details have been described previously [21]. Briefly, sera were incubated at $56^{\circ} \mathrm{C}$ for 30 minutes, centrifuged (13'000 rpm for 10 minutes), and diluted 1:8 in cell culture medium. Duplicates of five-fold serial dilutions were prepared in 96-well plates and mixed with 100 TCID50 of infectious SARS-CoV-2 in equal volume (BetaCoV/France/IDF0372/2020; National Reference Centre for Respiratory Viruses (Institut Pasteur, Paris, France). After incubation at $37^{\circ} \mathrm{C}$ for one hour, dilutions were transferred to confluent Vero E6 cells (provided by Prof. Dr. Volker Thiel, University of Bern, Bern, Switzerland) and incubated at $37^{\circ} \mathrm{C}$, $5 \% \mathrm{CO}_{2}$ and $>85 \%$ relative humidity for three days. $\mathrm{CPE}$ was assessed by crystal violet staining. Wells with an undisturbed cell layer were rated as (-), and signs of CPE were rated as (+). Full neutralization titer was defined as the serum dilution without signs of CPE (-) in both duplicates with a limit of detection (LOD) of $1: 16$. [21].

\section{Statistical analysis}

Descriptive statistics were used to describe the distribution of serological test results in individuals with and without COVID-19, overall and in subgroups. Diagnostic accuracy measures were calculated using predefined cut-offs of the serological index tests in relation to the reference standard as defined above (RT-PCR). Two-by-two tables were generated, sensitivities and specificities calculated, receiver-operating-characteristics (ROC) curves created, and c-statistics calculated. Serological test results were standardized using z-scores 
for test comparison in the context of the neutralization assay. [21]. Antibody response between subgroups was compared using unpaired t-test; P-values were reported without adjustment for multiple testing. As a power analysis, we used a method proposed by Bujang et al. [29]. We considered a prevalence of $6 \%$ (COVID-19) and a power of 0.8 to detect differences in specificity of 0.05 between assays. Analyses were carried out using the Stata 14.2 statistical software (StataCorp. 2014. Stata statistical software: Release 14. College Station, Tx: StataCorp LP). Figures were created using Prism 6 (GraphPad Software, Inc., LaJolla, California, United States).

\section{Patient and public involvement}

The study protocol, the questionnaire, and the first results have been presented on different occasions at Inselspital, and participant feedback was requested and incorporated.

\section{Results}

\section{Characteristics of participants}

Between April and November 2020, 3'658 consecutive individuals were included, comprising 1'085 inpatients with suspected SARS-CoV-2 infection and 2'573 medical personnel (Figure 1). Among those, 195 were COVID positive (prevalence 5.3\%). The median age was 46 years (standard deviation, SD, 16.2); $68 \%$ of the individuals were female (a high proportion of female nursing staff, in particular). The mean time since PCR was 59 days (SD 47). Detailed patient characteristics are given in Table 1.

\section{Diagnostic accuracy for the presence of previous COVID-19}

The distribution of results obtained with various serological tests in patients with and without previous COVID-19 is given in Figure 2; the respective cut-off levels are depicted as a grey line. The most significant overlap between COVID-19 positive and negative individuals was observed in the case of anti-N ELISA. In contrast, little overlap can be seen in anti-S1 ELISA and anti-N ECLIA.

The diagnostic accuracy of all tests in terms of receiver operating characteristics (ROC) curves is given in Figure 3. High area under the ROC curves (AUC) were observed for anti-S1 ELISA $(0.97 ; 95 \%$ confidence interval [CI] 0.95, 0.98), anti-RBD ELISA (0.95; 95\% CI 0.93, 0.97), anti-S1/S2 CLIA (0.95; 0.92, 0.97), and anti-N ECLIA $(0.94 ; 0.91,0.97)$. Lower AUC values were seen for anti-N ELISA $(0.90 ; 0.86,0.93)$ and anti-RBD+ LFI $(0.92 ; 0.88,0.95)$.

The differences in diagnostic accuracy of various serological tests are illustrated in Figure 6 (panel A); detailed results are given in Table 2. The sensitivity to detect patients with previous COVID-19 was [?]85\% in anti-N ECLIA $(86.8 \% ; 95 \%$ CI 81.1, 91.3) and anti-S1 ELISA $(86.2 \% ; 80.5,90.7)$, corresponding to 25 and 27 false-negative results. Sensitivity was $84.7 \%$ in anti-S1/S2 CLIA $(78.7,89.5), 84.0 \%$ in anti-RBD+ LFI $(76.6,89.8), 81.0 \%$ in anti-N CLIA $(74.6,86.3), 79.2 \%$ in anti-RBD ELISA $(72.7,84.7)$, and $65.6 \%$ in anti-N ELISA $(58.4,72.3)$. The corresponding numbers of false-negative results were 29 (anti-S1/S2 CLIA), 21 (anti-RBD+ LFI), 36 (anti-N CLIA), 40 (anti-RBD ELISA), and 66 (anti-N ELISA). Detailed diagnostic accuracy measures for all tests are given in Table 2.

The specificity was $98.4 \%$ in anti-N ECLIA $(98.0,98.8), 98.3 \%$ in anti-N CLIA $(97.8,98.7), 98.2 \%$ in anti-S1 ELISA (97.7, 98.6), 97.7\% in anti-N ELISA (97.2, 98.2), 97.6\% in anti-S1/S2 CLIA (97.0, 98.1), 97.2\% in anti-RBD ELISA $(96.5,97.7)$, and $96.1 \%$ in anti-RBD+ LFI $(95.3,96.9)$. The corresponding numbers of false-positive results were 54 (anti-N ECLIA), 60 (anti-N CLIA), 62 (anti-S1 ELISA), 79 (anti-N ELISA), 84 (anti-S1/S2 CLIA), 98 (anti-RBD ELISA), and 95 (anti-RBD+). 


\section{Diagnostic accuracy for the presence of neutralizing antibodies}

The accuracy of serological immunoassays for the presence of neutralizing antibodies was observed in a subgroup of complex patients $(\mathrm{n}=201)$. The association between the antibody response $(\mathrm{z}$-scored) and serum dilutions at full neutralization of live SARS-CoV-2 is depicted in Figure 4.

The differences in diagnostic accuracy of various serological tests are illustrated in Figure 6 (panel B); detailed results are given in Table 3 . The sensitivity to detect neutralizing antibodies was [?] $85 \%$ in anti-S1 ELISA $(92.7 \%$; 95\%CI 87.3, 96.3), anti-N ECLIA (91.7\%; 86.0, 95.6), anti-S1/S2 CLIA (90.3\%; 84.3, 94.6), anti-RBD+ LFI $(87.9 \% ; 80.3,93.4)$, and anti-RBD ELISA $(85.8 ; 91.0,79.1)$, corresponding to 11, 12, 14, 13, and 21 false-negative results, respectively. In contrast, sensitivity was $84.1 \%$ in anti-N CLIA $(77.2,89.7)$, and $66.2 \%$ in anti-N ELISA $(58.0,73.8)$. The corresponding numbers of false-negative results were 23 and 50 .

The specificity was [?] 97\% in anti-N CLIA (100\%; 91.8, 100), anti-S1/S2 CLIA (97.7\%; 87.7, 99.9), and antiRBD+ LFI $(97.9 \% ; 89.2,100)$, corresponding to 0,1 , and 1 false-positive results, respectively. Specificity was $95.9 \%$ in anti-RBD ELISA $(86.0,99.5), 93.0 \%$ in anti-N ECLIA $(80.9,98.5), 92 \%$ in anti-S1 ELISA $(80.8,97.8)$, and $65.3 \%$ in anti-N ELISA $(50.4,78.3)$. The corresponding numbers of false-positive results were $2,3,4$, and 17 respectively.

\section{Accuracy in salient subgroups of patients}

The antibody response in salient subgroups of patients is illustrated in Figure 5 (only COVID-19 positive individuals are shown). Significant higher antibody concentrations were observed in males, older individuals, inpatients, and patients admitted to intensive care units, including ventilated patients. As a sensitivity analysis, we calculated the diagnostic accuracy of the anti-S1 ELISA for the presence of COVID-19 in various subgroups (Table 4 ). The sensitivity was higher in males $(93.1 \%)$, probably reflecting more severe disease in this subgroup of patients. Other significant differences were not observed (Table 4).

\section{Biobank samples}

Analyzing 102 anonymized biobank samples collected from inpatients between December 2018 and February 2019, serological test results were negative in all samples in case of anti-S1 ELISA, anti-N ECLIA, and anti-S1/S2 CLIA. One positive test result was observed in the case of anti-RBD ELISA and anti-N CLIA $(1.0 \%)$. Four positive test results were observed in case of anti-N ELISA and anti-RBD+ LFI (3.9\%).

Discussion

We conducted a large prospective cross-sectional study in a real-life clinical setting stringently fulfilling the requirements of a diagnostic accuracy study and comparing all significant serological testing strategies. Sensitivities and specificities varied remarkably between different tests and were substantially different from manufacturer's specifications. The diagnostic accuracy in detecting patients with previous COVID-19 was high in anti-N ECLIA and anti-S1 ELISA (sensitivity [?] 85\%; specificity [?]97\%). The accuracy in detecting neutralizing antibodies was high in anti-S1/S2 CLIA and anti-RBD+ LFI (sensitivity [?] 85\%; specificity [?]97\%). Sensitivities and specificities obtained were consistent across various patient subgroups. With these diagnostic accuracy measures obtained in a real-life clinical setting, we were able to fill a critical gap in knowledge, identified by many previous authors, systematic reviews, and institutions such as the WHO $[2$, $5,9,10,20,30-32]$.

The study presented here adds important value as it was designed (1) as an adequately powered crosssectional study conducted in a real-life clinical setting, (2) to answer clearly defined clinical questions, (3) to include a representative study population, (3) to conduct a head-to-head comparison of all significant serological testing strategies, (4) to select and determine the reference standard test rigorously (e) apply 
optimal flow and timing. Specifically, we assessed whether different serological testing strategies may (a) accurately identify patients with previous COVID-19 and (b) correctly predict neutralizing antibodies against SARS-CoV-2. However, several potential limitations can be discussed. One might argue that we might have missed COVID-19 in some asymptomatic healthcare workers because individuals were asked to perform a nasopharyngeal swab in case of symptoms consistent with COVID-19. However, we do not believe that this might have affected the interpretation because it would not alter sensitivity and does not affect differences between different assays. Besides, a pre-specified complex subset of patients was selected for the live neutralization assay. This procedure ensures that there is no overestimation of performance.

The sensitivities and specificities obtained in clinical practice were considerably lower compared to previous publications. For example, the manufacturer of the anti-N ELICA claims a sensitivity of 100\% (Elecsys Anti-SARS-CoV-2 package insert; Roche diagnostics, Rotkreuz, Switzerland), and previous studies reported sensitivities between 96 and 100\% [33-38]. However, these study populations do not reflect real-life clinical practice, and the diagnostic accuracy measures can therefore not be applied to routine practice. In contrast, the sensitivity in our study, which was strictly designed to reflect clinical settings, was $87 \%$. These differences in sensitivity translate into a completely different interpretation of serological test results in seroprevalence studies and individual patients. Applying the sensitivity provided by the above-mentioned manufacturer (anti-N ECLIA) to our study population, the number of COVID-19 patients missed by the tests would be zero. In contrast, 25 COVID-19 patients were missed by the same test in our population (13.2\% of RTPCR positive individuals). The number of missed COVID-19 patients was even higher in other tests (e.g. anti-N ELISA; $\mathrm{n}=66 ; 34.3 \%$ ). Accordingly, the specificity of the anti-N ELICA was stated to be $99.8 \%$, corresponding to 7 patients falsely claimed to have had COVID-19 in our study cohort (false-positives). In contrast, we observed 54 false-positive individuals (falsely claimed to have COVID-19). These values are very similar in the case of the other serological tests.

The sensitivities and specificities must be taken into account to interpret test results correctly. We would like to give two examples to illustrate how this could be done. In a seroprevalence study in a setting similar to our study cohort, one can estimate the true prevalence by adding the numbers of false-negatives and subtracting the number of false-positives as calculated using the diagnostic accuracy measures determined from our study. Likewise, the probability of neutralizing antibodies in individual patients can be estimated in a similar calculation.

Our data support previous knowledge that the majority of patients with COVID-19 develop antibodies against epitopes of the SARS-CoV-2 and that these antibodies can be detected with a range of serological immunoassays. However, this does not apply to all patients and the clinical performance varies remarkably between different assays. A high diagnostic accuracy in terms of previous COVID-19 was observed for anti-N ECLIA and anti-S1 ELISA. In terms of neutralizing antibodies, the accuracy was high in anti-S1/S2 CLIA and anti-RBD+ LFI. Our data further confirm that the concentration of antibodies detected is strongly associated with the intensity of neutralizing antibodies, irrespective of assay technique. However, major questions remain which must be addressed in future studies: (1) can serological assays be used to distinguish between previous COVID-19 and vaccination, and (2) what is the accuracy of serological assays to predict protective immunity at certain serological cut-off levels.

\section{Conclusions}

In conclusions, sensitivities and specificities varied remarkably between different tests and were substantially lower than the manufacturer's specifications. These diagnostic accuracy measures can be used to calculate the virus burden within a specific population and determine the likelihood of protection against re-infection. Thus, our data might inform researchers, health professionals, and authorities to interpret seroprevalence studies and test results in individual patients. 


\section{Authorship details}

MPH, DB and AE developed the RBD immunoassay; MPH implemented and conducted all immunoassays. PB and FSR implemented and conducted the RT-PCR. HRJ and BW performed virus neutralization assays. MN developed the study design, the protocol, and the analysis plan, analyzed, interpreted the data, and wrote the manuscript. TF and CL developed the biobanking solutions. OE provided clinical data. LD and JM included patients. MF and FSR provided infrastructure, contributed to study design, and intellectually reviewed the manuscript. MPH, AE, BW, MF, and CL contributed to study design, interpretation of the results and drafting of the manuscript. All authors intellectually reviewed the manuscript. The corresponding author attests that all listed authors meet authorship criteria and that no others meeting the criteria have been omitted. The corresponding author affirms that the manuscript is an honest, accurate, and transparent account of the study being reported; no important aspects of the study have been omitted, and discrepancies from the study as originally planned have been explained.

\section{Funding/ Acknowledgements}

No particular funding was obtained for the purpose of this study. MN is supported by a research grant of the Swiss National Science Foundation (\#179334). AE received grant support from the Research Fund of the Swiss Lung Association, Bern and the Uniscientia foundation. The study was supported by a research grant of Buhlmann Laboratories (test kits). We thank Daniela Sturny, Christof Schild, Barbara Pula, Juliette Schlatter, Raphael Bratschi, Monika Hurni, Thomas Momot, Vincent Benites, Karin Volken, Margret Bachmann, Dominique Rowedder, Karin Balmer and Michelle Rickli for the great support. All authors, external and internal, had full access to all of the data in the study and can take responsibility for the integrity of the data and the accuracy of the data analysis.

\section{Declaration of interests}

The study was supported by a research grant of Buhlmann Laboratories (test kits). All authors declare that there is no conflict of interests. MN received research support from Roche diagnostics outside of the present work.

\section{Copyright/license for publication}

The Corresponding Author has the right to grant on behalf of all authors and does grant on behalf of all authors, a worldwide license to the Publishers and its licensees in perpetuity, in all forms, formats and media (whether known now or created in the future), to i) publish, reproduce, distribute, display and store the Contribution, ii) translate the Contribution into other languages, create adaptations, reprints, include within collections and create summaries, extracts and/or, abstracts of the Contribution, iii) create any other derivative work(s) based on the Contribution, iv) to exploit all subsidiary rights in the Contribution, v) the inclusion of electronic links from the Contribution to third party material where-ever it may be located; and, vi) licence any third party to do any or all of the above.

\section{Data sharing}

The study protocol can be requested from the corresponding author. The authors will make the relevant anonymized patient level data available on reasonable request. 


\section{References}

1. Riggioni C, Comberiati P, Giovannini M, Agache I, Akdis M, Alves-Correia M, et al. A compendium answering 150 questions on COVID-19 and SARS-CoV-2. Allergy. 2020;75(10):2503-41. Epub 2020/06/15. doi: 10.1111/all.14449. PubMed PMID: 32535955; PubMed Central PMCID: PMCPMC7323196.

2. Lisboa Bastos M, Tavaziva G, Abidi SK, Campbell JR, Haraoui LP, Johnston JC, et al. Diagnostic accuracy of serological tests for covid-19: systematic review and meta-analysis. BMJ. 2020;370:m2516. Epub 2020/07/03. doi: 10.1136/bmj.m2516. PubMed PMID: 32611558; PubMed Central PMCID: PMCPMC7327913 uniform disclosure form at www.icmje.org/coi_disclosure.pdf and declare: SL reports personal fees from Carebook Technologies, outside the submitted work; no financial relationships with any organisations that might have an interest in the submitted work in the previous three years; no other relationships or activities that could appear to have influenced the submitted work.

3. National S-C-SAEG. Performance characteristics of five immunoassays for SARS-CoV-2: a head-to-head benchmark comparison. Lancet Infect Dis. 2020;20(12):1390-400. Epub 2020/09/27. doi: 10.1016/S14733099(20)30634-4. PubMed PMID: 32979318; PubMed Central PMCID: PMCPMC7511171.

4. Winter AK, Hegde ST. The important role of serology for COVID-19 control. Lancet Infect Dis. 2020;20(7):758-9. Epub 2020/04/25. doi: 10.1016/S1473-3099(20)30322-4. PubMed PMID: 32330441; PubMed Central PMCID: PMCPMC7173803.

5. GeurtsvanKessel CH, Okba NMA, Igloi Z, Bogers S, Embregts CWE, Laksono BM, et al. An evaluation of COVID-19 serological assays informs future diagnostics and exposure assessment. Nat Commun. 2020;11(1):3436. Epub 2020/07/08. doi: 10.1038/s41467-020-17317-y. PubMed PMID: 32632160; PubMed Central PMCID: PMCPMC7338506.

6. Krammer F, Simon V. Serology assays to manage COVID-19. Science. 2020;368(6495):1060-1. Epub 2020/05/18. doi: 10.1126/science.abc1227. PubMed PMID: 32414781.

7. Bryant JE, Azman AS, Ferrari MJ, Arnold BF, Boni MF, Boum Y, et al. Serology for SARS-CoV-2: Apprehensions, opportunities, and the path forward. Sci Immunol. 2020;5(47). Epub 2020/05/21. doi: 10.1126/sciimmunol.abc6347. PubMed PMID: 32430309.

8. Tre-Hardy M, Blairon L, Wilmet A, Beukinga I, Malonne H, Dogne JM, et al. The role of serology for COVID-19 control: Population, kinetics and test performance do matter. J Infect. 2020;81(2):e91-e2. Epub 2020/05/18. doi: 10.1016/j.jinf.2020.05.019. PubMed PMID: 32417311; PubMed Central PMCID: PMCPMC7228733.

9. Peeling RW, Wedderburn CJ, Garcia PJ, Boeras D, Fongwen N, Nkengasong J, et al. Serology testing in the COVID-19 pandemic response. Lancet Infect Dis. 2020;20(9):e245-e9. Epub 2020/07/21. doi: 10.1016/S1473-3099(20)30517-X. PubMed PMID: 32687805; PubMed Central PMCID: PMCPMC7367660.

10. Oved K, Olmer L, Shemer-Avni Y, Wolf T, Supino-Rosin L, Prajgrod G, et al. Multi-center nationwide comparison of seven serology assays reveals a SARS-CoV-2 non-responding seronegative subpopulation. EClinicalMedicine. 2020;29:100651. Epub 2020/11/26. doi: 10.1016/j.eclinm.2020.100651. PubMed PMID: 33235985; PubMed Central PMCID: PMCPMC7676374.

11. Van Caeseele P, Canadian Public Health Laboratory N, Bailey D, Canadian Society of Clinical C, Forgie SE, Association of Medical M, et al. SARS-CoV-2 (COVID-19) serology: implications for clinical practice, laboratory medicine and public health. CMAJ. 2020;192(34):E973-E9. Epub 2020/08/06. doi: 10.1503/cmaj.201588. PubMed PMID: 32753391; PubMed Central PMCID: PMCPMC7840513 Public Health Laboratory (Manitoba Health). Mel Krajden has received grants paid to his institution from Roche, Hologica and Siemens. No other competing interests were declared. For competing interests of members of the 5 authorship groups, see Appendix 1, available at https://www.cmaj.ca/lookup/doi/10.1503/cmaj.201588/tab-related-content. 
12. Moshe M, Daunt A, Flower B, Simmons B, Brown JC, Frise R, et al. SARS-CoV-2 lateral flow assays for possible use in national covid-19 seroprevalence surveys (React 2): diagnostic accuracy study. BMJ. 2021;372:n423. Epub 2021/03/04. doi: 10.1136/bmj.n423. PubMed PMID: 33653694; PubMed Central PMCID: PMCPMC7921617 at www.icmje.org/coi_disclosure.pdf and declare: support from the Department of Health and Social Care, NIHR Biomedical Research Centre of Imperial College NHS Trust, UK Research and Innovation/Medical Research Council, Francis Crick Institute, Cancer Research UK, the Wellcome Trust, Huo Family Foundation and Imperial College London for the submitted work; PC, MM, and RT hold intellectual property rights on the hybrid DABA; no other relationships or activities that could appear to have influenced the submitted work.

13. Abbasi J. The Promise and Peril of Antibody Testing for COVID-19. JAMA. 2020;323(19):1881-3. Epub 2020/04/18. doi: 10.1001/jama.2020.6170. PubMed PMID: 32301958.

14. Chen X, Chen Z, Azman AS, Deng X, Sun R, Zhao Z, et al. Serological evidence of human infection with SARS-CoV-2: a systematic review and meta-analysis. Lancet Glob Health. 2021;9(5):e598-e609. Epub 2021/03/12. doi: 10.1016/S2214-109X(21)00026-7. PubMed PMID: 33705690; PubMed Central PMCID: PMCPMC8049592.

15. Gudbjartsson DF, Norddahl GL, Melsted P, Gunnarsdottir K, Holm H, Eythorsson E, et al. Humoral Immune Response to SARS-CoV-2 in Iceland. N Engl J Med. 2020;383(18):1724-34. Epub 2020/09/02. doi: 10.1056/NEJMoa2026116. PubMed PMID: 32871063; PubMed Central PMCID: PMCPMC7494247.

16. Ismail AA. Serological tests for COVID-19 antibodies: Limitations must be recognized. Ann Clin Biochem. 2020;57(4):274-6. Epub 2020/04/29. doi: 10.1177/0004563220927053. PubMed PMID: 32343598.

17. Shuren J, Stenzel T. The FDA's Experience with Covid-19 Antibody Tests. N Engl J Med. 2021;384(7):592-4. Epub 2021/02/20. doi: 10.1056/NEJMp2033687. PubMed PMID: 33605568.

18. Castells MC, Phillips EJ. Maintaining Safety with SARS-CoV-2 Vaccines. N Engl J Med. 2021;384(7):643-9. Epub 2020/12/31. doi: 10.1056/NEJMra2035343. PubMed PMID: 33378605; PubMed Central PMCID: PMCPMC7787218.

19. Duong YT, Wright CG, Justman J. Antibody testing for coronavirus disease 2019: not ready for prime time. BMJ. 2020;370:m2655. Epub 2020/07/06. doi: 10.1136/bmj.m2655. PubMed PMID: 32620675; PubMed Central PMCID: PMCPMC7332962 ties to commercial companies. The authors declare the following other interests: none. The BMJ policy on financial interests is here: https://www.bmj.com/sites/default/files/attachments/resources/2016/03/16-current- bmj-educationcoi-form.pdf.

20. Andrey DO, Cohen P, Meyer B, Torriani G, Yerly S, Mazza L, et al. Head-to-Head Accuracy Comparison of Three Commercial COVID-19 IgM/IgG Serology Rapid Tests. J Clin Med. 2020;9(8). Epub 2020/07/30. doi: 10.3390/jcm9082369. PubMed PMID: 32722191; PubMed Central PMCID: PMCPMC7463984.

21. Brigger D, Horn MP, Pennington LF, Powell AE, Siegrist D, Weber B, et al. Accuracy of serological testing for SARS-CoV-2 antibodies: First results of a large mixed-method evaluation study. Allergy. 2021;76(3):853-65. Epub 2020/10/01. doi: 10.1111/all.14608. PubMed PMID: 32997812; PubMed Central PMCID: PMCPMC7537154.

22. Wolfensberger N, Georgiou G, Giabbani E, Reusser M, Njue LM, Fiedler M, et al. Rapid Centrifugation in the Routine Hemostasis Laboratory. Thromb Haemost. 2019;119(12):2025-33. Epub 2019/10/11. doi: 10.1055/s-0039-1696712. PubMed PMID: 31600807.

23. Dahlweid FM, Kaempf M, Leichtle A. Interoperability of laboratory data in Switzerland - a spotlight on Bern. Laboratoriumsmedizin. 2018;42:251—8.

24. Brosi L, Kubler E, Weston A, Romann P, Panikulam S, Dirscherl L, et al. Development of a Unique Rapid Test to Detect Anti-bodies Directed Against an Extended RBD of SARS-CoV-2 Spike Protein. Chimia 
(Aarau). 2021;75(5):446-52. Epub 2021/05/22. doi: 10.2533/chimia.2021.446. PubMed PMID: 34016243.

25. Lippi G, Salvagno GL, Pegoraro M, Militello V, Caloi C, Peretti A, et al. Preliminary evaluation of Roche Cobas Elecsys Anti-SARS-CoV-2 chemiluminescence immunoassay. Clin Chem Lab Med. 2020;58(11):e251e3. Epub 2020/07/22. doi: 10.1515/cclm-2020-1042. PubMed PMID: 32692698.

26. Tang MS, Hock KG, Logsdon NM, Hayes JE, Gronowski AM, Anderson NW, et al. Clinical Performance of Two SARS-CoV-2 Serologic Assays. Clin Chem. 2020;66(8):1055-62. Epub 2020/05/14. doi: 10.1093/clinchem/hvaa120. PubMed PMID: 32402061; PubMed Central PMCID: PMCPMC7239232.

27. Bonelli F, Sarasini A, Zierold C, Calleri M, Bonetti A, Vismara C, et al. Clinical and Analytical Performance of an Automated Serological Test That Identifies S1/S2-Neutralizing IgG in COVID-19 Patients Semiquantitatively. J Clin Microbiol. 2020;58(9). Epub 2020/06/26. doi: 10.1128/JCM.01224-20. PubMed PMID: 32580948; PubMed Central PMCID: PMCPMC7448652.

28. Corman VM, Landt O, Kaiser M, Molenkamp R, Meijer A, Chu DK, et al. Detection of 2019 novel coronavirus (2019-nCoV) by real-time RT-PCR. Euro Surveill. 2020;25(3). Epub 2020/01/30. doi: 10.2807/15607917.ES.2020.25.3.2000045. PubMed PMID: 31992387; PubMed Central PMCID: PMCPMC6988269.

29. Bujang MA, Adnan TH. Requirements for Minimum Sample Size for Sensitivity and Specificity Analysis. J Clin Diagn Res. 2016;10(10):YE01-YE6. Epub 2016/11/29. doi: 10.7860/JCDR/2016/18129.8744. PubMed PMID: 27891446; PubMed Central PMCID: PMCPMC5121784.

30. Mekonnen D, Mengist HM, Derbie A, Nibret E, Munshea A, He H, et al. Diagnostic accuracy of serological tests and kinetics of severe acute respiratory syndrome coronavirus 2 antibody: A systematic review and meta-analysis. Rev Med Virol. 2021;31(3):e2181. Epub 2020/11/06. doi: 10.1002/rmv.2181. PubMed PMID: 33152146.

31. Caini S, Bellerba F, Corso F, Diaz-Basabe A, Natoli G, Paget J, et al. Meta-analysis of diagnostic performance of serological tests for SARS-CoV-2 antibodies up to 25 April 2020 and public health implications. Euro Surveill. 2020;25(23). Epub 2020/06/20. doi: 10.2807/1560-7917.ES.2020.25.23.2000980. PubMed PMID: 32553061; PubMed Central PMCID: PMCPMC7403641.

32. Vengesai A, Midzi H, Kasambala M, Mutandadzi H, Mduluza-Jokonya TL, Rusakaniko S, et al. A systematic and meta-analysis review on the diagnostic accuracy of antibodies in the serological diagnosis of COVID-19. Syst Rev. 2021;10(1):155. Epub 2021/05/28. doi: 10.1186/s13643-021-01689-3. PubMed PMID: 34039423; PubMed Central PMCID: PMCPMC8152206.

33. Egger M, Bundschuh C, Wiesinger K, Gabriel C, Clodi M, Mueller T, et al. Comparison of the Elecsys(R) Anti-SARS-CoV-2 immunoassay with the EDI enzyme linked immunosorbent assays for the detection of SARS-CoV-2 antibodies in human plasma. Clin Chim Acta. 2020;509:18-21. Epub 2020/06/03. doi: 10.1016/j.cca.2020.05.049. PubMed PMID: 32485155; PubMed Central PMCID: PMCPMC7261064.

34. Wakita M, Idei M, Saito K, Horiuchi Y, Yamatani K, Ishikawa S, et al. Comparison of the clinical performance and usefulness of five SARS-CoV-2 antibody tests. PLoS One. 2021;16(2):e0246536. Epub 2021/02/09. doi: 10.1371/journal.pone.0246536. PubMed PMID: 33556086; PubMed Central PMCID: PMCPMC7870088.

35. Chan CW, Parker K, Tesic V, Baldwin A, Tang NY, van Wijk XMR, et al. Analytical and Clinical Evaluation of the Automated Elecsys Anti-SARS-CoV-2 Antibody Assay on the Roche cobas e602 Analyzer. Am J Clin Pathol. 2020;154(5):620-6. Epub 2020/08/21. doi: 10.1093/ajcp/aqaa155. PubMed PMID: 32814955; PubMed Central PMCID: PMCPMC7454296.

36. Muench P, Jochum S, Wenderoth V, Ofenloch-Haehnle B, Hombach M, Strobl M, et al. Development and Validation of the Elecsys Anti-SARS-CoV-2 Immunoassay as a Highly Specific Tool for Determining Past Exposure to SARS-CoV-2. J Clin Microbiol. 2020;58(10). Epub 2020/08/05. doi: 10.1128/JCM.01694-20. PubMed PMID: 32747400; PubMed Central PMCID: PMCPMC7512151. 
37. Perez-Garcia F, Perez-Tanoira R, Iglesias ME, Romanyk J, Arroyo T, Gomez-Herruz P, et al. Comparative evaluation of six immunoassays for the detection of antibodies against SARS-CoV-2. J Virol Methods. 2021;289:114047. Epub 2020/12/19. doi: 10.1016/j.jviromet.2020.114047. PubMed PMID: 33338544; PubMed Central PMCID: PMCPMC7832212.

38. Manthei DM, Whalen JF, Schroeder LF, Sinay AM, Li SH, Valdez R, et al. Differences in Performance Characteristics Among Four High-Throughput Assays for the Detection of Antibodies Against SARS-CoV-2 Using a Common Set of Patient Samples. Am J Clin Pathol. 2021;155(2):267-79. Epub 2020/10/10. doi: 10.1093/ajcp/aqaa200. PubMed PMID: 33033840; PubMed Central PMCID: PMCPMC7665294.

\section{Tables}

Table 1: Patient characteristics. Consecutive inpatients and medical personnel with suspected COVID-19 were included in a prospective cross-sectional study in a real-life clinical setting ( $\left.\mathrm{n}=3^{\prime} 658\right)$.

\begin{tabular}{llll}
\hline & No. (\%) & No. (\%) & No. (\%) \\
\hline Characteristic & All & COVID-19 positive & COVID-19 negative \\
No. (\%) & $3^{\prime} 658(100)$ & $195(5.3)$ & $3^{\prime} 463(94.7)$ \\
Age, mean (SD), years & $46.0(16.2)$ & $46.9(17.0)$ & $45.9(16.2)$ \\
Female gender & $2^{\prime} 478(68.4)$ & $108(55.4)$ & $2^{\prime} 370(69.1)$ \\
Inpatients & $1^{\prime} 085(29.7)$ & $94(48.2)$ & $991(28.6)$ \\
Medical personnel & $2^{\prime} 573(70.3)$ & $101(51.8)$ & $2^{\prime} 472(71.4)$ \\
Days since PCR, mean (SD), days & $58.6(47.0)$ & $46.0(48.6)$ & $60.3(46.5)$ \\
Hospitalization & $1^{\prime} 085(29.7)$ & $94(48.2)^{*}$ & $991(28.6)^{*}$ \\
ICU & N/A\# & $32(16.4)$ & N/A\# \\
Mechanical ventilation & N/A & $26(13.3)$ & N/A\# \\
No symptoms & N/A & $27(13.8)$ & N/A\# \\
Fever & N/A\# & $94(48.2)$ & N/A\# \\
\hline
\end{tabular}

* Corresponding to respective number of inpatients; \# information not available in COVID-19 negative inpatients

Table 2: Diagnostic accuracy of various serological immunoassays for the presence of previous COVID-19. Results of a diagnostic accuracy study are shown.

\begin{tabular}{|c|c|c|c|c|c|c|c|}
\hline $\begin{array}{l}\text { Analytical } \\
\text { tech- } \\
\text { nique }\end{array}$ & ELISA & ELISA & ELISA & ECLIA & CLIA & CLIA & LFI \\
\hline Epitope & $\begin{array}{l}\text { Anti- } \\
\text { RBD }\end{array}$ & Anti-S1 & Anti-N & Anti-N & Anti-N & $\begin{array}{l}\text { Anti- } \\
\text { S1/S2 }\end{array}$ & $\begin{array}{l}\text { Anti- } \\
\text { RBD+ }\end{array}$ \\
\hline $\begin{array}{l}\text { Antibody } \\
\text { subtype }\end{array}$ & IgG & $\operatorname{IgG}$ & IgG & Pan-Ig & IgG & IgG & Pan-Ig \\
\hline \multicolumn{2}{|c|}{ ManufacturerIn-house } & Euroimmun & $\begin{array}{l}\text { Epitope } \\
\text { diagnos- } \\
\text { tics }\end{array}$ & $\begin{array}{l}\text { Roche } \\
\text { diagnos- } \\
\text { tics }\end{array}$ & Abbott & DiaSorin & Bühlmann \\
\hline $\begin{array}{l}\text { Numbers } \\
\text { of patients }\end{array}$ & 3'637 & 3’658 & $3 ’ 654$ & $3 ’ 630$ & 3'630 & 3'630 & $2^{\prime} 589$ \\
\hline $\begin{array}{l}\text { True } \\
\text { positives }\end{array}$ & 152 & 168 & 126 & 164 & 153 & 160 & 110 \\
\hline
\end{tabular}




\begin{tabular}{|c|c|c|c|c|c|c|c|}
\hline $\begin{array}{l}\text { Analytical } \\
\text { tech- } \\
\text { nique }\end{array}$ & ELISA & ELISA & ELISA & ECLIA & CLIA & CLIA & LFI \\
\hline $\begin{array}{l}\text { False } \\
\text { negatives }\end{array}$ & 40 & 27 & 66 & 25 & 36 & 29 & 21 \\
\hline $\begin{array}{l}\text { False } \\
\text { positives }\end{array}$ & 98 & 62 & 79 & 54 & 60 & 84 & 95 \\
\hline $\begin{array}{l}\text { True } \\
\text { negatives }\end{array}$ & $3^{\prime} 347$ & 3'401 & 3’383 & $3 ’ 387$ & $3 ’ 381$ & $3 ’ 357$ & $2 ’ 363$ \\
\hline $\begin{array}{l}\text { AUC }(95 \% \\
C I)\end{array}$ & $\begin{array}{l}0.95(0.93 \\
0.97)\end{array}$ & $\begin{array}{l}0.97(0.95 \\
0.98)\end{array}$ & $\begin{array}{l}0.90(0.86 \\
0.93)\end{array}$ & $\begin{array}{l}0.94(0.91 \\
0.97)\end{array}$ & $\begin{array}{l}0.95(0.93, \\
0.98)\end{array}$ & $\begin{array}{l}0.95(0.92 \\
0.97)\end{array}$ & $\begin{array}{l}0.92(0.88 \\
0.95)\end{array}$ \\
\hline $\begin{array}{l}\text { Sensitivity } \\
\text { (percent; } \\
95 \% C I \text { ) }\end{array}$ & $\begin{array}{l}79.2(72.7 \\
84.7)\end{array}$ & $\begin{array}{l}86.2(80.5 \\
90.7)\end{array}$ & $\begin{array}{l}65.6(58.4 \\
72.3)\end{array}$ & $\begin{array}{l}86.8 \text { (81.1, } \\
91.3)\end{array}$ & $\begin{array}{l}81.0(74.6, \\
86.3)\end{array}$ & $\begin{array}{l}84.7(78.7, \\
89.5)\end{array}$ & $\begin{array}{l}84.0(76.6, \\
89.8)\end{array}$ \\
\hline $\begin{array}{l}\text { Specificity } \\
\text { (percent; } \\
95 \% \text { CI) }\end{array}$ & $\begin{array}{l}97.2(96.5, \\
97.7)\end{array}$ & $\begin{array}{l}98.2 \text { (97.7, } \\
98.6)\end{array}$ & $\begin{array}{l}97.7 \text { (97.2, } \\
98.2)\end{array}$ & $\begin{array}{l}98.4(98.0, \\
98.8)\end{array}$ & $\begin{array}{l}98.3(97.8, \\
98.7)\end{array}$ & $\begin{array}{l}97.6(97.0, \\
98.1)\end{array}$ & $\begin{array}{l}96.1 \text { (95.3, } \\
96.9)\end{array}$ \\
\hline $\begin{array}{l}\text { Positive } \\
\text { likelihood } \\
\text { ratio }\end{array}$ & 28 & 48 & 29 & 55 & 46 & 35 & 22 \\
\hline $\begin{array}{l}\text { Negative } \\
\text { likelihood } \\
\text { ratio }\end{array}$ & 0.21 & 0.41 & 0.35 & 0.13 & 0.19 & 0.16 & 0.17 \\
\hline
\end{tabular}

Consecutive inpatients and medical personnel with suspected COVID-19 were included in a prospective crosssectional study in a real-life clinical setting $\left(\mathrm{n}=3^{\prime} 658\right)$. A positive RT-PCR from a nasopharyngeal swab was defined as the primary reference standard test representing a "previous SARS-CoV-2" infection (COVID-19). Abbreviations: ELISA, enzyme-linked immunosorbent assay; ECLIA, electrochemiluminescence immunoassay; CLIA, chemiluminescence immunoassay; LFI, lateral flow immunoassay; N, nucleoprotein; RBD, receptor binding domain of the SARS-CoV-2 spike glycoprotein; S1/2, domain 1 or 2 of the SARS-CoV-2 spike glycoprotein; RBD+, extended receptor binding domain of the SARS-CoV-2 spike glycoprotein

Table 3: Diagnostic accuracy of serological immunoassays for the presence of neutralizing antibodies.

\begin{tabular}{llllll}
\hline Analytical technique & ELISA & ELISA & ELISA & ECLIA & CLIA \\
\hline Epitope & Anti-RBD & Anti-S1 & Anti-N & Anti-N & Anti- \\
Antibody subtype & IgG & IgG & IgG & IgG/IgM & IgG \\
Manufacturer & In-house & Euroimmun & Epitope diagnostics & Roche diagnostics & Abbo \\
Numbers of patients & 197 & 201 & 197 & 188 & 188 \\
True positives & 127 & 140 & 98 & 133 & 122 \\
False negatives & 21 & 11 & 50 & 3 & 23 \\
False positives & 2 & 4 & 17 & 40 & 0 \\
True negatives & 47 & 46 & 32 & $91.7(86.0,95.6)$ & $84.1(7)$ \\
Sensitivity (percent; $95 \% C I)$ & $85.8(91.0,79.1)$ & $92.7(87.3,96.3)$ & $66.2(58.0,73.8)$ & $93.0(80.9,98.5)$ & $100(9$ \\
Specificity (percent; 95\%CI) & $95.9(86.0,99.5)$ & $92.0(80.8,97.8)$ & $65.3(50.4,78.3)$ & & \\
\hline
\end{tabular}

A subset of complex patients was selected for analysis using the live SARS-CoV-2 neutralization assay because of limited resources $(n=201)$. Abbreviations: ELISA, enzyme-linked immunosorbent assay; ECLIA, electrochemiluminescence immunoassay; CLIA, chemiluminescence immunoassay; LFI, lateral flow immunoassay; 
$\mathrm{N}$, nucleoprotein; RBD, receptor binding domain of the SARS-CoV-2 spike glycoprotein; S1/2, domain 1 or 2 of the SARS-CoV-2 spike glycoprotein; RBD+, extended receptor binding domain of the SARS-CoV-2 spike glycoprotein

Table 4: Diagnostic accuracy of an anti-S1 ELISA ( $\operatorname{IgG}$ ) for the presence of previous COVID-19 in various subgroups. Results of a diagnostic accuracy study are given $\left(n=3^{\prime} 637\right)$.

\begin{tabular}{|c|c|c|c|c|c|}
\hline Subgroup & Numbers of patients & True positives & False negatives & False positives & True negativ \\
\hline & No. & No. & No. & No. & No. \\
\hline \multicolumn{6}{|c|}{ Patient population } \\
\hline Full study cohort & 3'658 & 168 & 27 & 62 & 3'401 \\
\hline Inpatients & 1'085 & 80 & 14 & 15 & 976 \\
\hline Outpatients* & $2^{\prime} 573$ & 88 & 13 & 47 & $2^{\prime} 438$ \\
\hline \multicolumn{6}{|l|}{ Gender } \\
\hline Male & 1'147 & 81 & 6 & 18 & 1’042 \\
\hline Female & $2^{\prime} 478$ & 87 & 21 & 43 & $2^{\prime} 348$ \\
\hline Age & & $\mathrm{s}$ & & & \\
\hline$<50$ years & 2'201 & 89 & 17 & 42 & 2'070 \\
\hline$>50$ years & $1^{\prime} 457$ & 79 & 10 & 20 & 1'348 \\
\hline \multicolumn{6}{|l|}{ Symptoms } \\
\hline Any symptom & $1^{\prime} 985$ & 143 & 25 & 39 & $1^{\prime} 751$ \\
\hline No symptoms & 2'473 & 88 & 13 & 47 & $2 ' 425$ \\
\hline \multicolumn{6}{|l|}{ Days since PCR } \\
\hline 28 & 3'116 & 96 & 14 & 55 & 2’951 \\
\hline 21 & 3'205 & 107 & 15 & 56 & 3’027 \\
\hline 14 & 3’304 & 128 & 15 & 57 & 3'104 \\
\hline
\end{tabular}

\section{Figure legends}

Figure 1: Flowchart illustrating the study design and procedures of a prospective crosssectional study in a real-life clinical setting (diagnostic accuracy study). Consecutive health-care workers and inpatients with suspected or possible previous COVID-19 were prospectively included. Abbreviations: ELISA, enzyme-linked immunosorbent assay; ECLIA, electrochemiluminescence immunoassay; CLIA, chemiluminescence immunoassay; LFI, lateral flow immunoassay; N, nucleoprotein; RBD, receptor binding domain of the SARS-CoV-2 spike glycoprotein; S1/2, domain 1 or 2 of the SARS-CoV-2 spike glycoprotein; RBD+, extended receptor binding domain of the SARS-CoV-2 spike glycoprotein; RT-PCR, real-time PCR.

Figure 2: Distribution of serological testing results in patients with and without previous COVID-19. IgG or pan-Ig responses against the nucleoprotein $(\mathrm{N})$, the receptor binding domain of the SARS-CoV-2 spike glycoprotein (RBD), the domain 1 or 2 of the SARS-CoV-2 spike glycoprotein (S1/S2), and the extended RBD domain are shown. Consecutive health-care workers and inpatients with suspected or possible previous COVID-19 were prospectively included ( $\mathrm{n}=3{ }^{\prime} 658$; prevalence $5.3 \%$ ).

Figure 3: Accuracy of various serological tests for the identification of patients with previous COVID-19. Receiver operating characteristics (ROC) curves of IgG or pan-Ig responses against the nucleoprotein $(\mathrm{N})$, the receptor binding domain of the SARS-CoV-2 spike glycoprotein (RBD), the domain 1 or 2 of the SARS-CoV-2 spike glycoprotein (S1/S2), and the extended RBD domain are shown. Consecutive health-care workers and inpatients with suspected or possible previous COVID-19 were prospectively included ( $\mathrm{n}=3$ '658; prevalence $5.3 \%$ ). 
Figure 4: Antibody responses and live SARS-CoV-2 neutralization. Z-scored serological test results and serum dilutions at full neutralization are shown as determined in 201 selected individuals (nonlinear curve fitting). Abbreviations: ELISA, enzyme-linked immunosorbent assay; ECLIA, electrochemiluminescence immunoassay; CLIA, chemiluminescence immunoassay; LFI, lateral flow immunoassay; N, nucleoprotein; RBD, receptor binding domain of the SARS-CoV-2 spike glycoprotein; S1/2, domain 1 or 2 of the SARS-CoV-2 spike glycoprotein; RBD+, extended receptor binding domain of the SARS-CoV-2 spike glycoprotein

Figure 5: Antibody response in salient subgroups of patients. Anti-S1 ELISA results in patients with previous COVID-19 are given. ${ }^{*} \mathrm{P}=0.0005 ; * * \mathrm{P}<0.0001$

Figure 6: Comparative diagnostic accuracy of various serological tests for (A) the identification of patients with previous COVID-19, and (B) the presence of neutralizing antibodies. Abbreviations: ELISA, enzyme-linked immunosorbent assay; ECLIA, electrochemiluminescence immunoassay; CLIA, chemiluminescence immunoassay; LFI, lateral flow immunoassay; N, nucleoprotein; RBD, receptor binding domain of the SARS-CoV-2 spike glycoprotein; S1/2, domain 1 or 2 of the SARS-CoV-2 spike glycoprotein; $\mathrm{RBD}+$, extended receptor binding domain of the SARS-CoV-2 spike glycoprotein

\section{Hosted file}

allFigures.pdf available at https://authorea.com/users/428955/articles/532737-serologicaltesting-for-sars-cov-2-antibodies-in-clinical-practice-a-comparative-diagnosticaccuracy-study 